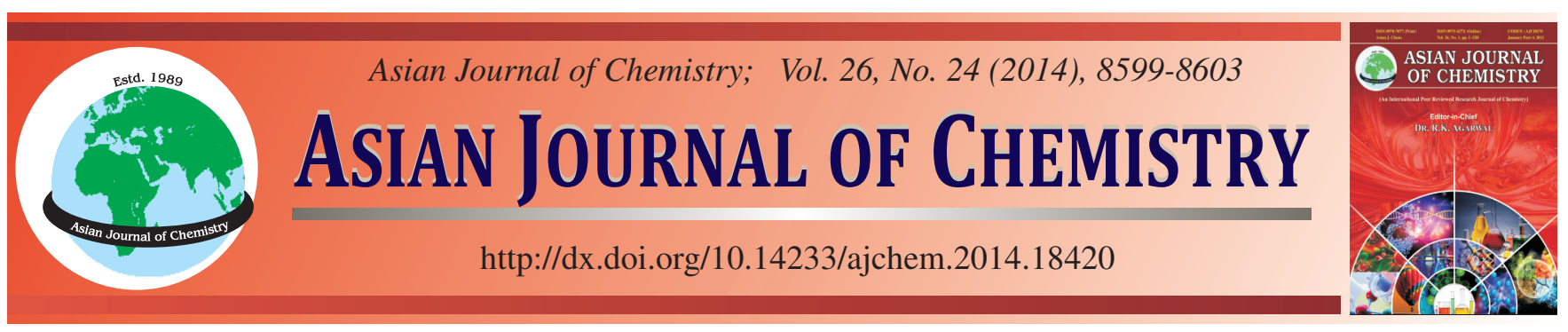

\title{
Eco-Friendly and Sensitive Simultaneous Determination of Unbound Hydrochlorothiazide and Cefditoren Pivoxil in Human Plasma Using High Performance Frontal Analysis
}

\author{
H.M. Albishri
}

Chemistry Department, Faculty of Science, King Abdulaziz University, 80203 Jeddah, Saudi Arabia

Corresponding author: E-mail: hmalbeshri@kau.edu.sa

\begin{abstract}
Eco-friendly and sensitive simultaneous determination of unbound hydrochlorothiazide and cefditoren pivoxil in human plasma was developed by a direct injection high-performance frontal analysis. The high-performance frontal analysis method consists of an highperformance frontal analysis column with a mobile phase of $67 \mathrm{mM}$ phosphate buffer $(\mathrm{pH} 7.4$ ) at flow rate of $0.2 \mathrm{~mL} / \mathrm{min}$ and wavelength $260 \mathrm{~nm}$. The injection volume of plasma, which is a crucial factor in high-performance frontal analysis, was determined to be $1000 \mu \mathrm{L}$. The injection of $10 \mu \mathrm{L}$ sample eluted drugs as typical HPLC peaks with retention times after the protein peak. The high-performance frontal analysis method was validated according to FDA guideline for Industry-Bioanalytical Method Validation. The linearity of unbound hydrochlorothiazide and cefditoren pivoxil was achieved in the range of 1-200 and 0.3-200 ng/mL with limits of quantifications of 1.1 and $7.1 \mathrm{ng} / \mathrm{mL}$, respectively. Adequate accuracy, precision and stability were obtained. This study illustrates how high-performance frontal analysis could be used for eco-friendly determination of unbound drug as a function of drug pharmacokinetic parameters with great advantages of its sensitivity and ease of its operation.
\end{abstract}

Keywords: Frontal analysis, Cefditoren pivoxil, Hydrochlothiazide, Plasma.

\section{INTRODUCTION}

The extent of plasma protein binding is considered one of the important physiological factors affecting pharmacokinetic characteristics and the pharmacological efficacy of a drug. When a drug is administered, it enters the blood stream and is bound to plasma proteins ${ }^{1}$. Unbound drug in the plasma transfers freely to the target organ, whereas bound drug hardly passes through the blood capillary walls to reach the action site. Some important pharmacokinetic properties such as hepatic metabolism rate, renal excretion rate, biomembrane permeation rate and steady-state distribution volume are the function of the unbound $\mathrm{drug}^{2}$. In order to determine unbound drug amount, equilibrium dialysis and ultrafiltration were commonly used. However, these conventional methods involved problems such as drug adsorption onto the membrane and leakage of strongly bound drug through the membrane ${ }^{2}$.

To overcome these difficulties, high-performance frontal analysis (HPFA) was developed and used for the sensitive analysis of unbound drug ${ }^{1-6}$. In HPFA, it was used a restricted access column which excludes macromolecules of plasma proteins but retains a drug of small molecular size. An excess volume of plasma sample contained drug is directly injected into an HPFA column under a mild mobile phase condition (physiological pH 7.4 of phosphate buffer without adding any organic modifier). Then, the release of bound drug from protein is apparently suppressed and the chromatographic partition equilibrium inside the micropores concurrently with the drugprotein binding equilibrium in the interstices was established ${ }^{2}$. The drug concentration in the stagnant flow of mobile phase consequently becomes the same as the unbound drug concentration and consequently the same as the drug concentration in the injected sample. After that, drug and protein are separated from each other with elution of unbound drug as a trapezoidal peak having a plateau region. The plateau height is used to calculate the unbound drug concentration. During the separation, the drug-protein binding equilibrium is kept constant because plasma protein binding is a reversible and kinetically rapid process. The analysis by HPFA methodology has several advantages over conventional methods that it allows direct sample injection analysis without any pretreatment process and eco-friendly without using of any organic solvents.

Recently, combined chemotherapy is becoming popular due to the fact that many diseases require multiple agents to be used in complex regimens. Hydrochlorothiazide is a thiazide diuretic that is indicated as a first line therapy for the treatment of hypertension and heart failure in many countries. Hydrochlorothiazide is not metabolized but is eliminated rapidly by the kidney. Peak plasma concentration of hydrochlorothiazide is small (70-490 ng/mL) within 1 to $5 \mathrm{~h}$ of dosing $25 \mathrm{mg}^{7}$. The 
most common combination products in the treatment of hypertension comprise a thiazide diuretic plus several categories. The mechanism of action of diuretics is still controversial, but there is increased retention of sodium by the hypertensive kidney when blood pressure is lowered by non-diuretic drugs, thus reducing antihypertensive efficacy. Some antibacterial drugs such as cefditoren pivoxil (CFP, the most common) combined with hydrochlorothiazide have already been described ${ }^{8}$.

The analysis of hydrochlorothiazide and cefditoren pivoxil in human plasma is an important criterion. The complexity of plasma matrix adds challenge to direct determination of drugs without sample pretreatment. There are some liquid chromatographic (LC) reports for the determination of cefditoren pivoxil ${ }^{9-11}$ and hydrochlorothiazide ${ }^{12-15}$ as an individual or combined with other drugs. These analytical methodologies were involved extraction steps or expensive and easy-to-contaminate detectors (e.g., mass spectrometry). In addition, the using of toxic organic solvents is a common factor in all methods. Recently, the (bio)pharmaceutical industry introduced safety and health regulations to protect the environmental criteria for their products ${ }^{16}$. The problem is further elaborated by a report from Glaxo Smith Kline (GSK) ${ }^{17}$ which estimated that $80 \%$ of the waste is coming out from organic solvents. This suggests that (bio)pharmaceutical industry will contribute dramatically to alleviating this environmental problem. Recently, we developed an eco-friendly cyclodextrine modified micellar liquid chromatography method for the total drug concentration of hydrochlorothiazide and cefditoren pivoxil in plasma ${ }^{8}$. There is no any report about the determination of unbound hydrochlorothiazide and cefditoren pivoxil in human plasma. Therefore, in the present work, an eco-friendly and sensitive HPFA method was developed for the simultaneous determination of unbound hydrochlorothiazide and cefditoren pivoxil in human plasma. The monitoring of their unbound concentrations is of clinical importance because of their low therapeutic plasma concentration and their strong binding with plasma protein.

\section{EXPERIMENTAL}

Hydrochlorothiazide (HCZ, $\geq 99 \%$ ) was purchased from Sigma (St. Louis, MO, USA). Cefditoren pivoxil (CFP, $\geq 99.5$ $\%$ ) was obtained from local pharmaceutical laboratory (Jeddah, Saudi Arabia). Potassium dihydrogen phosphate, sodium hydroxide and orthophosphoric acid were purchased from Sigma (St. Louis, MO, USA). All of the reagents were used without further purifications. Human plasma samples were collected from healthy volunteers (males, 27-30 years old) and were shaken at $37^{\circ} \mathrm{C}$ for $30 \mathrm{~min}$ before analysis. Water used for the preparations of both solutions and mobile phases was purified by a Milli-Rx apparatus (Millipore, Milford, MA, USA).

The HPFA measurements was carried out by PerkinElmer series 200 LC binary solvent delivery system (San Diego, Canada) with a Rheodyne injection valve [model 7725(i), $2 \mathrm{~mL}$ loop], a series $200 \mathrm{UV}=$ visible variable wavelength Detector and series 200 vacuum degasser. Data was collected with Total Chrom Chromatography Data Handling System. The PerkinElmer 600 series Link Interface was used with software to acquire and buffer digital data from the instrument and to control the operating parameter of such instrument. The temperature of column was maintained constant at $37^{\circ} \mathrm{C}$ using a column oven (Model 200, PerkinElmer, Canada). An ISRP (internal surface reversed phase) HPFA column $(150 \mathrm{~mm} \times$ $4.6 \mathrm{~mm}$ I.D., $5 \mu \mathrm{m}$ ) was obtained from Regis Chemical Company (Morton Grove, IL). A small ISRP security guard precolumn was incorporated into the system. The flow rate was $0.2 \mathrm{~mL} /$ min. The detection wavelength was $260 \mathrm{~nm}$ which is suitable for both drugs. The MiniTab software package (USA statistical software) was employed to perform the statistical analysis of data.

Preparation of solutions: Stock standard solutions of hydrochlorothiazide and cefditoren pivoxil were accurately prepared in a $10 \mathrm{~mL}$ measuring flask by dissolving $25 \mathrm{mg}$ of both in phosphate buffer. The stock standard solution was transferred into PTFE-sealed screw-cap bottles and stored at $4{ }^{\circ} \mathrm{C}$ until the required analysis. Working solutions were prepared daily by appropriate dilution from stock standard solution in the same solvent. Phosphate buffer solution $(67 \mathrm{mmol} / \mathrm{L})$ was prepared by weighing an appropriate amount of potassium dihydrogen phosphate and adjusted to $\mathrm{pH} 7.4$ using sodium hydroxide.

Method validation: The high-performance frontal analysis method was validated according to most recent FDA Guidelines for Industry Bioanalytical Method Validation by validation of the following assay-specific parameters 18: selectivity, linearity, limit of detection (LOD), limit of quantitation (LOQ), accuracy, intra- and inter-days precision and stability of analytes in the plasma matrices.

The selectivity of analytes was determined by the measurement of 10 samples collected from healthy controls. Firstly, the samples were determined if there are any co-eluting peaks which may interfere with the target compounds. Secondly, the samples were spiked with the limits of quantification of analytes to determine if any co-eluting peaks could be reported as a presumptive positive.

Linearity was studied by preparing standard working solutions of the studied drugs to obtain the required concentrations. For obtaining the calibration graphs, plateau heights (unbound drug concentration) were plotted against the corresponding analyte concentrations $(\mathrm{ng} / \mathrm{mL})$. Limit of detection (LOD) was calculated by the equation: $\mathrm{LOD}=3.3 \mathrm{\sigma} / \mathrm{S}$, where $\sigma$ is the standard deviation of response of blank injection and $\mathrm{S}$ is the slope of the calibration plot. Limit of quantification (LOQ) was calculated from LOQ $=10 \mathrm{\sigma} / \mathrm{S}$.

Precision, accuracy and stability of analytes were determined using plasma based quality control samples prepared in drug-free plasma from healthy controls. Three replicates from each sample were measured by three separate runs in order to assess precision. Stability of analytes was evaluated by comparing the plateau height of working standard samples with those obtained for samples subjected to stability tests. Biological samples were stored frozen at $-20{ }^{\circ} \mathrm{C}$ for 30 days to check the long-term stability studies but during short-term (bench-top) stability samples were kept at room conditions for $24 \mathrm{~h}$. The stability after three freeze-thaw cycles was tested.

Determination of the unbound hydrochlorothiazide and cefditoren pivoxil in human plasma: Human plasma samples $(5 \mathrm{~mL})$ were collected from healthy men and appropriate amounts of hydrochlorothiazide and cefditoren pivoxil was added. Spiked samples were vigorously shaken and 
incubated at $37{ }^{\circ} \mathrm{C}$ for $30 \mathrm{~min}$ and then filtered by using Millipore filters. An aliquot $(1000 \mu \mathrm{L})$ was directly injected into the HPFA column by the injector-reswitching technique at the proper time ${ }^{4-5,19-20}$ with a mobile phase of phosphate buffer $(\mathrm{pH} 7.4,67 \mathrm{mM})$ without any organic additives so as not to disturb the protein binding equilibrium. Flow rate was fixed at $0.2 \mathrm{~mL} / \mathrm{min}$ during the analysis time in order to prevent any clogging of the column by an irreversible adsorption of the protein $^{21}$. Before pumping to the LC system, the contents of the mobile phase were filtered through $0.45 \mu \mathrm{m}$ membrane filter and degassed by applying ultrasonic vibrations for 10 min. Each measurement was repeated in duplicate to give precise measurements. A blank experiment was carried out simultaneously.

\section{RESULTS AND DISCUSSION}

High-performance frontal analysis (HPFA) method optimization: The most commonly used stationary phase in HPFA is restricted access internal surface reversed phase (ISRP) materials ${ }^{19,21}$. Internal surface reversed phase column of $150 \mathrm{~mm} \times 4.6 \mathrm{~mm}$ I.D. and $5 \mu \mathrm{m}$ at physiological $\mathrm{pH}(\mathrm{pH}$ 7.4) of phosphate buffer (67 mM, physiological concentration) as mobile phase without addition of any organic modifier was used. Because of the plasma concentration of hydrochlorothiazide is small, a long HPFA column $(150 \mathrm{~mm})$ was selected for this assay to extract large volumes of unbound drugs. This type of columns consists of a hydrophilic external surface covered with diol-glycine and a hydrophobic internal (micropore) surface containing diol-glycyl-phenylalanylphenylalanine. A small hydrophobic analyte, such as hydrochlorothiazide and cefditoren pivoxil, can penetrate into the micropore and is retained on the hydrophobic ligand, while plasma protein is size excluded. To suppress any undesirable peak diffusion in the extra column dead volume, the LC system was modified as much as possible ${ }^{21,22}$. A piece of small-bore PEEK tubing $(25 \mathrm{~cm} \times 0.005$ inch I.D.) was used to connect the injector valve to the column. A graphite filter was connected to the HPFA column by use of a direct column coupler. The column outlet was directly connected to the inlet SUS tubing (0.2 mm I.D.) of a micro flow-cell of the UV detector. However, there is a problem concerning the durability of the column: the column pressure increased by three-fold after 10 analyses of the plasma sample. Therefore, special care should be taken in order to prevent an increase in the column pressure in case of plasma sample analyses. Using a guard column was useful for this purpose.

It is essential to obtain a plateau region, for which the sample injection volume should be properly optimized. If the plasma injection volume is insufficient, drugs will be released from protein and eluted as typical HPLC peaks with retention times as like as the reference peaks and after the protein peak (Fig. 1). Addition of an organic modifier into the mobile phase serves for the quick release of bound drug and improves the peak shape. Fig. 2 shows the effect of acetonitrile addition in the mobile phase on the HPFA separation of hydrochlorothiazide and cefditoren pivoxil in human plasma. When a small volume $(10 \mu \mathrm{L})$ of analytes-human plasma solution is directly injected to HPFA column, the sample solution is diluted with mobile phase, resulting in complete release of the bound drugs.

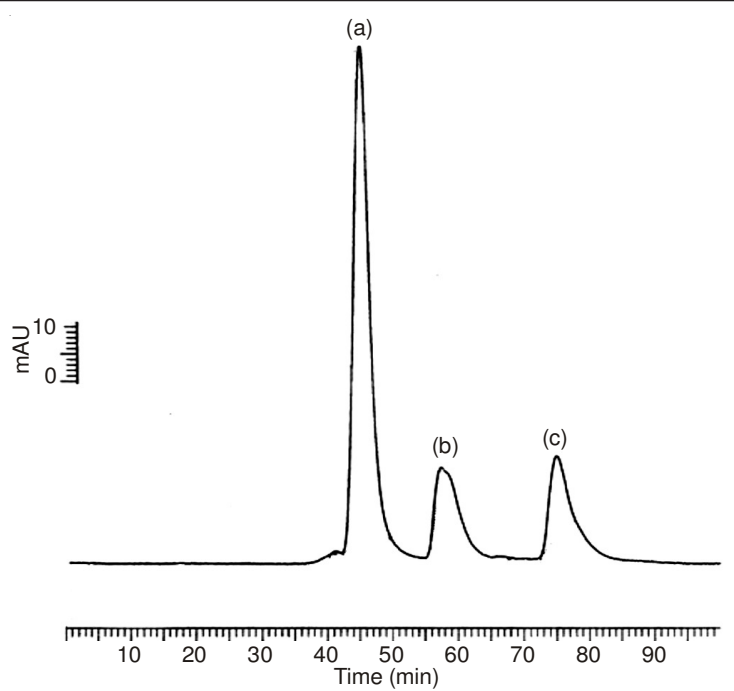

Fig. 1. High-performance frontal analysis chromatogram for the simultaneous determination of $4 \mathrm{ng} / \mathrm{mL}$ of hydrochlorothiazide (b) and 25 $\mathrm{ng} / \mathrm{mL}$ of cefditoren pivoxil (c) in human plasma (a for human proteins) under the following conditions: HPFA column (ISRP, 150 $\mathrm{mm} \times 4.6 \mathrm{~mm}$ I.D., $5 \mu \mathrm{m})$; mobile phase: $67 \mathrm{mM}$ phosphate buffer at $\mathrm{pH} 7.4$; injection volume: $10 \mu \mathrm{L}$; Flow rate: $0.2 \mathrm{~mL} / \mathrm{min}$; temperature: $37^{\circ} \mathrm{C}$ and wavelength: $260 \mathrm{~nm}$

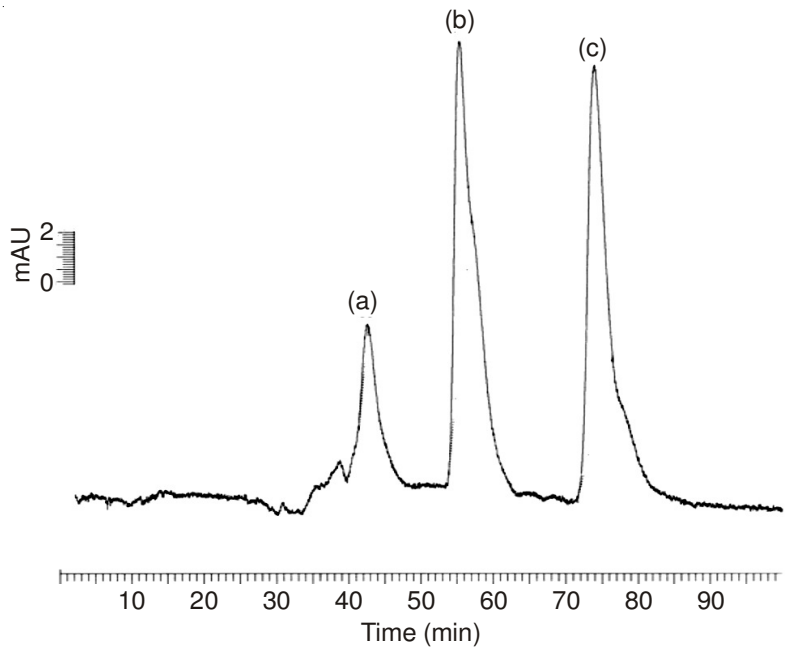

Fig. 2. High-performance frontal analysis chromatogram for the simultaneous determination of $10 \mathrm{ng} / \mathrm{mL}$ of hydrochlorothiazide (a) and $50 \mathrm{ng} / \mathrm{mL}$ of cefditoren pivoxil (b) in human plasma (a) for human proteins) using mobile phase of $67 \mathrm{mM}$ phosphate buffer:acetonitrile (15:85 \%, v/v). Other conditions as cited in Fig. 1

Thus, total drugs (bound+unbound) were retained on column and were eluted out of the column to give resolved sharp peaks (Fig. 2).

As the plasma injection volume is increased drugs will reach the same protein binding equilibrium as in the initial plasma sample and a plateau region will form. Fig. 3 shows the HPFA chromatograms in the absence of any organic solvents in the mobile phase after the injection of high volumes of human plasma solutions contained hydrochlorothiazide alone (Fig. 3A), cefditoren pivoxil alone (Fig. 3B) and ( $\mathrm{HCZ}+$ CFP) mixture (Fig. 3C). The HPFA injection volume was optimized for hydrochlorothiazide and cefditoren pivoxil to be $1000 \mu \mathrm{L}$. The injection volume, after which the plateau height did not increase and only broadened the plateau region, was reported as the minimum injection volume $(\mathrm{MIV})^{20}$. The 
simultaneous HPFA analyses for the unbound hydrochlorothiazide and cefditoren pivoxil in human plasma were completed within $10 \mathrm{~min}$ analysis time as indicated in Fig. 3C. Furthermore, due to the slow kinetic interactions between drugs and plasma proteins, the time of incubation for the samples was studied in the period range from 0 to $60 \mathrm{~min}$. It was found that the optimal incubation time under $37{ }^{\circ} \mathrm{C}$ give the best signals of measurements is $30 \mathrm{~min}$. After optimization of the most effective parameters in HPFA analysis, it is crucial now to validate the proposed method.

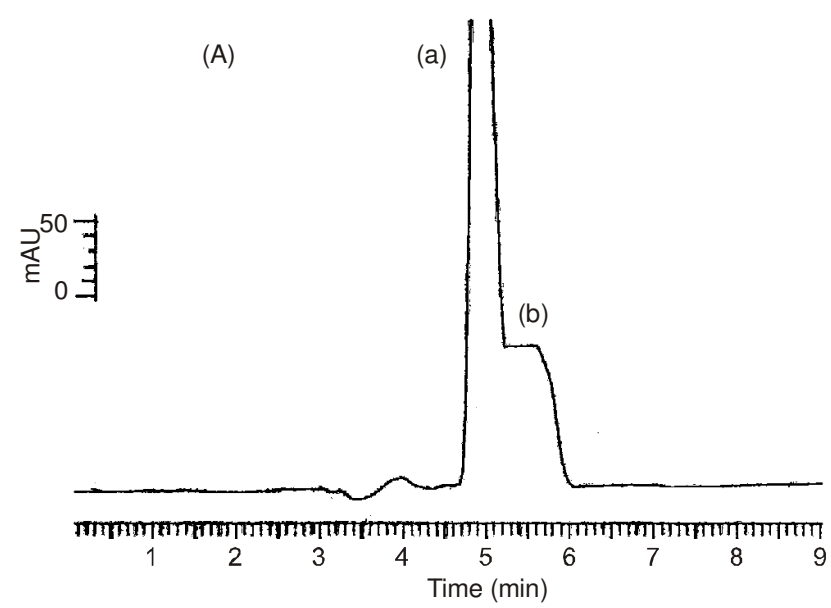

(B)

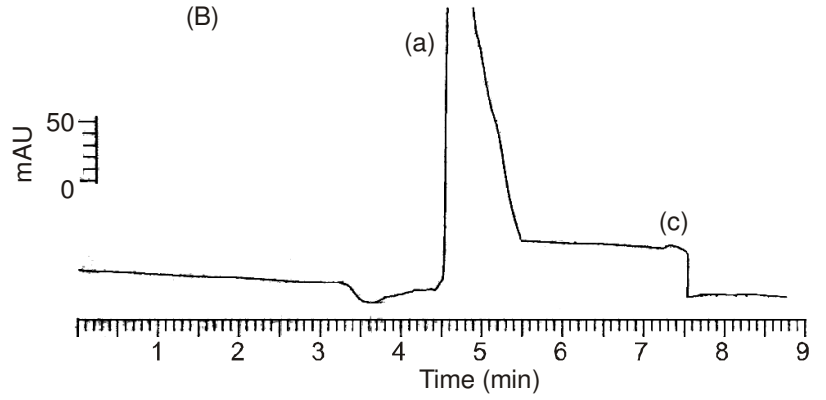

(C)

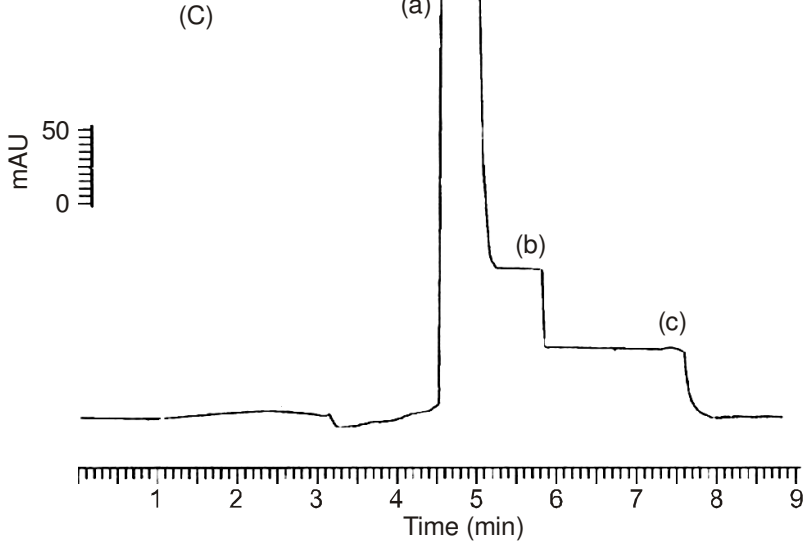

Fig. 3. High-performance frontal analysis chromatograms after the injection of high plasma volumes $(1000 \mu \mathrm{L})$ contained $100 \mathrm{ng} / \mathrm{mL}$ of hydrochlorothiazide (b) in (A) or $100 \mathrm{ng} / \mathrm{mL}$ of cefditoren pivoxil (c) in (B) or $100 \mathrm{ng} / \mathrm{mL}$ of (HCZ (b) +CFP (c)) in (C) in the presence of human proteins (peak a) under the experimental conditions as shown in Fig. 1

Method validation: The selectivity of the proposed HPFA system for the determination of hydrochlorothiazide and cefditoren pivoxil in human plasma was studied. Drugs-free plasma samples were selected as blanks and processed directly in the chromatographic system. They were then analyzed to determine the extent to which endogenous components may contribute to interfere with retention time of the drugs after spiking. It was found that no interference from proteins and other compounds in human plasma on the unbound analytes measurements.

The linearity of the dependence of peak height on unbound drug concentration was verified by triplicate analyses of seven standard solutions containing 1-200 and 7-200 ng/mL of hydrochlorothiazide and cefditoren pivoxil, respectively and injected directly to HPFA system. The least square linear regression analysis of the data gave the following equations:

Peak height $=0.58+0.05$ concentration $(\mathrm{ng} / \mathrm{mL}), \mathrm{r}=0.998$ for hydrochlorothiazide

Peak height $=0.95+0.09$ concentration $(\mathrm{ng} / \mathrm{mL}), \mathrm{r}=0.996$ for cefditoren pivoxil

Statistical analysis of the data gave small values of the standard deviations (SDs) of 0.68 and 0.59 for hydrochlorothiazide and cefditoren pivoxil, respectively. Limit of quantitation (LOQ) and limit of detection (LOD) in human plasma were calculated as described before. Limits of quantification values were found to be of 1.1 and $7.1 \mathrm{ng} / \mathrm{mL}$, while limit of detection values were found to be 0.4 and $2.2 \mathrm{ng} / \mathrm{mL}$ for hydrochlorothiazide and cefditoren pivoxil, respectively.

The accuracy was determined by applying the optimized HPFA with three injection replicates at three concentration levels covering the linearity range of both analytes. The obtained mean recoveries were ranged from 93.3 to $106.7 \%$. Precision tests were studied to determine both intra-day and inter-day variations in peak height. The obtained relative standard deviations (RSDs, $n=5$ ) were reported in Table-1. These results provided an indication of the applicability of HPFA for routine analytical estimations.

Stability of the HPFA method for the determination of unbound analytes in human plasma was evaluated by comparing the peak height or concentration of the low, medium and high concentration levels of working plasma samples with those subjected to stability tests at ambient temperature over $24 \mathrm{~h}$ (bench top stability) or samples were stored frozen at $-20{ }^{\circ} \mathrm{C}$ for 30 days (long term stability) or the stability after three freeze-thaw cycles. Table- 2 indicates the stability studies at the low, medium and high concentration levels of the studied drugs under varying conditions. The obtained recovery values also proved the stability of HPFA conditions.

In order to demonstrate the applicability of the proposed HPFA method, several aliquots of hydrochlorothiazide and cefditoren pivoxil authentic solutions were added into plasma samples as described. No peaks of hydrochlorothiazide and cefditoren pivoxil metabolites appeared because these compounds could not be detected within 10 min run time ${ }^{8}$. A further confirmation of the accuracy of the determination was obtained by evaluating the level of hydrochlorothiazide or cefditoren pivoxil in plasma samples, by means of interpolation with the standard addition curve. The found data were in agreement with those obtained with the single point calibration method as no significant differences were observed by applying the F- and t-test at the $95 \%$ confidence level. In order to determine the unbound hydrochlorothiazide or cefditoren pivoxil concentrations, human plasma samples spiked with $100 \mathrm{ng} / \mathrm{mL}$ of hydrochlorothiazide or cefditoren pivoxil were pretreated as 


\begin{tabular}{ccccc}
\hline \multicolumn{5}{c}{ TABLE-1 } \\
& \multicolumn{5}{c}{ REPLICATE ANALYSIS AND ACCURACY OF HYDROCHLOROTHIAZIDE AND } \\
& CEFDITOREN PIVOXIL IN HUMAN PLASMA BY HIGH-PERFORMANCE FRONTAL ANALYSIS \\
\hline Compound & Unbound concentration $(\mathrm{ng} / \mathrm{mL})$ & Found concentration $(\mathrm{ng} / \mathrm{mL})$ & Recovery $(\%)$ & RSD $(\%)(\mathrm{n}=5)$ \\
\hline \multirow{3}{*}{ Hydrochlorothiazide } & 1.5 & 1.4 & 93.3 & 1.1 \\
& 50.0 & 49.1 & 98.2 & 0.8 \\
\hline \multirow{3}{*}{ Cefditoren pivoxil } & 150.0 & 150.0 & 100.0 & 0.5 \\
& 7.5 & 7.6 & 101.3 & 0.9 \\
& 50.0 & 50.0 & 100.0 & 0.7 \\
\end{tabular}

\begin{tabular}{|c|c|c|c|c|c|c|}
\hline \multicolumn{7}{|c|}{$\begin{array}{c}\text { TABLE-2 } \\
\text { STABILITY STUDIES OF HYDROCHLOROTHIAZIDE AND } \\
\text { CEFDITOREN PIVOXIL IN HUMAN PLASMA UNDER VARYING CONDITIONS }\end{array}$} \\
\hline \multirow{3}{*}{ Parameter } & \multicolumn{6}{|c|}{ Actual unbound concentration $(\mathrm{ng} / \mathrm{mL})$} \\
\hline & \multicolumn{3}{|c|}{ Hydrochlorothiazide } & \multicolumn{3}{|c|}{ Cefditoren pivoxil } \\
\hline & 1.5 & 50.0 & 150.0 & 7.5 & 50.0 & 150.0 \\
\hline \multicolumn{7}{|c|}{ (a) Bench top stability } \\
\hline Found concentration $(\mathrm{ng} / \mathrm{mL})$ & 1.6 & 50.0 & 150.1 & 7.6 & 50.1 & 149.9 \\
\hline $\operatorname{RSD}(\%), \mathrm{n}=5$ & 0.9 & 0.7 & 0.5 & 0.8 & 0.6 & 0.5 \\
\hline Recovery $(\%)$ & 106.7 & 100.0 & 100.1 & 101.3 & 100.2 & 99.9 \\
\hline \multicolumn{7}{|c|}{ (b) Re-injection after 30 days at $-20{ }^{\circ} \mathrm{C}$} \\
\hline Found concentration $(\mathrm{ng} / \mathrm{mL})$ & 1.4 & 49.7 & 150.1 & 7.5 & 50.0 & 150.1 \\
\hline $\operatorname{RSD}(\%), \mathrm{n}=5$ & 0.8 & 0.5 & 0.7 & 0.3 & 0.5 & 0.6 \\
\hline Recovery $(\%)$ & 93.3 & 99.4 & 100.1 & 100.0 & 100.0 & 100.1 \\
\hline \multicolumn{7}{|c|}{ (c) Three freeze-thaw cycles } \\
\hline Found concentration $(\mathrm{ng} / \mathrm{mL})$ & 1.4 & 50.2 & 150.2 & 7.4 & 49.8 & 150.0 \\
\hline $\operatorname{RSD}(\%), \mathrm{n}=5$ & 0.9 & 0.7 & 0.6 & 0.9 & 0.6 & 0.4 \\
\hline Recovery $(\%)$ & 93.3 & 100.4 & 100.1 & 98.7 & 99.6 & 100.0 \\
\hline
\end{tabular}

explained in the experimental section and then directly injected to HPFA system. The unbound hydrochlorothiazide and cefditoren pivoxil concentrations were determined and found to be $15.2 \pm 1.4$ and $45.5 \pm 2.8 \mathrm{ng} / \mathrm{mL}$, respectively $(\mathrm{n}=5)$.

\section{Conclusion}

An eco-friendly and sensitive HPFA method for simultaneous determination of unbound hydrochlorothiazide and cefditoren pivoxil in human plasma was successfully developed for the first time. One advantage of the HPFA procedure compared to the other reports is the direct injection of human plasma samples without any organic solvent additives for the unbound drug concentration with adequate precise and accurate measurements. Both drugs were successfully determined in the level of $\mathrm{ng} / \mathrm{mL}$ in human plasma. As well, the proposed method meets the requirements of the "green chemistry" concept with a relatively inexpensive tool and the absence of toxic solvents. Therefore, HPFA has a possibility for high-throughput bioanalysis of combined chemotherapies, thus making it more attractive.

\section{ACKNOWLEDGEMENTS}

The authors are greatly thankful for the financial support of our work by the funding of King Abdulaziz University, Jeddah, Saudi Arabia.

\section{REFERENCES}

1. A. Shibukawa, T. Nakagawa, A. Kaihara, K. Yagi and H. Tanaka, Anal. Chem., 59, 2496 (1987).

2. A. Shibukawa, Y. Kuroda and T. Nakagawa, Trends Analyt. Chem., 18, 549 (1999).
3. A. Shibukawa, T. Nakagawa, N. Nishimura, M. Miyake and H. Tanaka, Chem. Pharm. Bull. (Tokyo), 37, 702 (1989).

4. A. Shibukawa, M. Nagao, Y. Kuroda and T. Nakagawa, Anal. Chem., 62, 712 (1990).

5. N. Nishimura, A. Shibukawa and T. Nakagawa, Anal. Sci., 6, 355 (1990).

6. A. Shibukawa, A. Terakita, J. He and T. Nakagawa, J. Pharm. Sci., 81, 710 (1992).

7. http://dailymed.nlm.nih.gov/dailymed/lookup.cfmisetid=238176e5df10-457b-a5cf-c950a583e748, 15 June (2014).

8. D. Abd El-Hady and H.M. Albishri, J. Liq. Chromatogr., 37, 2681 (2014).

9. W. Rieck and D. Platt, Clin. Lab., 46, 477 (2000).

10. R.V. Nirogi, V.N. Kandikere, W. Shrivastava and K. Mudigonda, Arzneimittelforschung, 56, 309 (2006).

11. S.R. Narala and K. Saraswathi, J. Pharm. Sci. Res., 3, 1002 (2011).

12. D.V. Bharathi, K.K. Hotha, P.K. Chatki, V. Satyanarayana and V. Venkateswarlu, Bioanal., 4, 1195 (2012).

13. A.G. Jangid, R.H. Tale and V.V. Vaidya, Biomed. Chromatogr., 26, 95 (2012).

14. R.N. Sharma and S.S. Pancholi, Acta Pharm., 62, 45 (2012).

15. S. Kurbanoglu, M. Gumustas and S.A. Ozkan, J. Pharm. Biomed. Anal., 72, 198 (2013).

16. H.M. Albishri, D. Abd El-Hady and R.A. Tayeb, Acta Chromatogr; 10.1556/AChrom.27.2015.3.5.

17. A.D. Curzons, C. Jimenez-Gonzalez, A.L. Duncan, D.J.C. Constable and V.L. Cunningham, Int. J. Life Cycle Assess., 12, 272 (2007).

18. CDER, Center for Drug Evaluation and Research. Guidance for Industry Bioanalytical Method Validation; www.fda.gov/downloads/ Drugs/GuidanceComplianceRegulatoryInformation/Guidances/ UCM070107.pdf (2001).

19. F. Li, D. Zhou and X. Guo, J. Chromatogr. Sci., 41, 137 (2003).

20. N. Amini, R. Nakao, M. Schou and C. Halldin, J. Pharm. Biomed. Anal., 98, 140 (2014).

21. T. Kimura, K. Nakanishi, T. Nakagawa, A. Shibukawa and K. Matsuzaki, J. Pharm. Biomed. Anal., 38, 204 (2005).

22. A. Terakita, A. Shibukawa, C. Nakao and T. Nakagawa, Anal. Sci., 10, 11 (1994). 